\title{
Comunicación gubernamental pre-covid de los presidentes hispanohablantes en Twitter
}

\author{
Pre-covid goverment communication \\ of spanish-speaking presidents on Twitter
}

\author{
Manuel Antonio Conde \\ Universidad de Huelva (España) \\ manuelantonioconde@gmail.com \\ https://orcid.org/0000-0002-7382-2166 \\ Oscar A. Prada-Espinel \\ Universidad de Cartagena (Colombia) \\ oprada9@gmail.com \\ https://orcid.org/0000-0002-2974-9504 \\ Kevin P. Pullaguari-Zaruma \\ Universidad Técnica Particular de Loja - UTPL (Ecuador) \\ kppullaguari@utpl.edu.ec \\ https://orcid.org/0000-0001-6546-2618
}

\begin{abstract}
Resumen
El presente trabajo analiza la comunicación gubernamental y competencia mediática de los presidentes de los países hispanohablantes previa a la declaración de pandemia por coronavirus el 11 de marzo de 2020 por la OMS. Una declaración sin precedentes en la historia, pero que los medios de comunicación desde las semanas previas ya estaban informando en sus cuentas de Twitter. El objetivo general fue analizar la comunicación gubernamental y competencia mediática que tuvieron los presidentes de los países hispanohablantes la semana previa a la declaración de pandemia por COVID-19, y para ello elegimos una investigación cuantitativa, ya que esta nos permitió un análisis de datos con un método flexible. Mediante la minería con algoritmos de búsqueda avanzada de datos en Twitter analizamos 7650 tuits de las cuentas de los 22 presidentes y 21 medios de comunicación, estableciendo seis parámetros de medida de las dimensiones de la competencia mediática: índice Flesch-Kincaid, engagement, soporte audiovisual, índice link-hashtag, índice de autenticidad y la agenda building. A pesar de los indicadores de la OMS y los medios de comunicación, llegamos a la conclusión de que muchos de los presidentes no tuvieron una comunicación política y competencia mediática aceptable acorde con el problema abordado.
\end{abstract}

\section{Palabras clave}

Comunicación gubernamental, competencia mediática, Twitter, hispanohablantes, coronavirus, Covid-19.

Forma sugerida de citar: Conde, M., Prada-Espinel, O., \& Pullaguari-Zaruma, K. (2021). Comunicación gubernamental pre-covid de los presidentes hispanohablantes en Twitter. Universitas, 34, pp. 63-86. 


\begin{abstract}
This work analyzes the goverment communication and media competence of the presidents of spanishspeaking countries prior to the declaration of a coronavirus pandemic on March 11, 2020 by the WHO. A statement unprecedented in history, but that the media from the previous weeks were already reporting on their Twitter accounts. The general objective was to analyze the government communication and media competence that the presidents of the spanish-speaking countries had the week prior to the declaration of a COVID-19 pandemic, and for this we chose a quantitative research, since this allowed us to analyze data with a method flexible. By mining with advanced data search algorithms on Twitter, we analyzed 7650 tweets from the accounts of the 22 presidents and 21 media outlets, establishing six measurement parameters of the dimensions of media competence: Flesch-Kincaid index, engagement, audiovisual support, link-hashtag index, authenticity index and the building agenda. Despite the WHO and media indicators, we concluded that many of the presidents did not have acceptable political communication and media competence commensurate with the problem addressed.
\end{abstract}

\title{
Keywords
}

Government communication, media competence, Twitter, spanish-speakers, coronavirus, Covid-19.

\section{Introducción}

\section{De la comunicación gubernamental a la comunicación en tiempos de crisis}

A nivel general la comunicación política es aquella que estudia las dimensiones contradictorias y complementarias que se presentan en la política, información y comunicación en sistemas democráticos (Reyes et al., 2011). De igual manera, la interacción y transmisión de mensajes por parte de partidos políticos y ciudadanos o bien entre gobernantes y gobernados (Reyes et al., 2011). La comunicación política presenta múltiples matices que en ocasiones tienden a confundirse entre sí, por lo que resulta imprescindible aclarar que las principales formas en que se puede presentar son comunicación gubernamental, electoral, de crisis y de riesgo (Riorda, 2011). Para este esfuerzo investigativo nos enfocaremos en la comunicación gubernamental y la comunicación de crisis. 
La comunicación gubernamental, para Canel y Sanders (2011), se refiere a los objetivos, el papel y las prácticas comunicativas implementadas por los políticos, ejecutivos y funcionarios de instituciones públicas al servicio de una justificación política, que se constituye basándose en el consentimiento directo o indirecto de la ciudadanía, entre los que se encuentran grupos sociales, empresariales, minorías, etc., que buscan influir en el gobierno para formular e implementar políticas beneficiosas para la sociedad en general, apoyándose en el marketing, los mass media, las redes sociales y otros instrumentos que actualmente ofrece la web. En otras palabras, es el proceso comunicativo gubernamental que busca garantizar una cohesión, funcionamiento y diversificación de la información para con partidos políticos, ciudadanía y el sistema político global (Molina, 2004).

El fin más importante de la comunicación gubernamental en una sociedad democrática es, en teoría, mostrar que el líder electo y su gabinete cumplen con los planes de gobernanza propuestos en candidatura, como también la defensa de los intereses gubernamentales y los intereses del estado por encima de los particulares, bajo la premisa de la construcción de una sociedad equitativa haciendo los procesos de la agenda pública transparentes; sobre todo, en momentos de tensión y crisis, cuya definición para nosotros es: un suceso o un conjunto de estos que perturban, dificultan o ponen en riesgo la normalidad y el desarrollo de un determinado proceso o estado en la esfera social que repercute directamente en el cuerpo social y todo lo que le subyace (sector público y privado, relaciones interpersonales, economía, gobernabilidad, etc.).

Una crisis, en primera instancia, genera una sensación de incertidumbre, de ignorancia respecto a la dirección que tomarán los acontecimientos; en segundo lugar, surge la necesidad de intervenir, seleccionar las medidas correctas y decidir cómo aplicarlas lo antes posible (Bauman \& Bordoni, 2016). El manejo de esta necesita orientación basada en la evidencia para la comunicación de crisis (Coombs, 2007), que debe ser normativa, tal como lo es su gestión (Heath, 2010).

Para abordar una crisis se deben considerar aspectos propios de un contexto, los cuales son sus fortalezas y debilidades. Existen tres fases genéricas:

- La precrisis: se recopila la información sobre los riegos que acrecientan el problema, esto con el fin de entenderlo, prepararse y afrontarlo (Coombs, 2010). 
- Crisis: basándose en la recopilación se tomarán decisiones y se crearán mensajes para las personas (Coombs, 2010), acordes a la problemática que se afronta. Es decir, busca dar respuesta a la crisis de manera efectiva.

- La poscrisis: implica diseccionar el esfuerzo de gestión de crisis, comunicar los cambios precisos a la ciudadanía y proporcionar mensajes de seguimiento de la crisis según sea necesario (Coombs, 2010), para la prevención de problemáticas similares a futuro.

Para Coombs (2010), la comunicación de crisis es la búsqueda de la información, su tratamiento y transmisión para abordar con éxito una situación difícil, cuya respuesta comunicativa debe estar definida por el modo en que sienten los afectados, lo que precisan conocer, lo que se les puede o no decir o hacer y por las posibilidades en que se debe de ayudar (Bonilla et al., 2013). Por consiguiente, es muy importante tener expertos examinando los datos resultantes de la interacción ciudadana mediante las redes sociales - no será la totalidad de la población, pero sí una proporción importantebuscar patrones comunes, hacer una recopilación de lo expresado negativa y positivamente; en algunos casos, según requiera el manejo de la crisis, segmentar por regiones teniendo en cuenta sus necesidades propias y aspectos climáticos, sociales y culturales de cada una.

Por ello, se deben establecer estrategias de respuesta a la crisis para reducir el efecto negativo y prevenir intenciones de comportamiento nocivas e incluso para reparar la reputación (Coombs, 2007) de la esfera gubernamental. También, se deben abrir nuevos canales de comunicación para tomar de manera ordenada y coordinada las acciones comunicativas adecuadas que merece la situación.

Sin embargo, las respuestas ante una crisis son variables debido a que no todas se abordan ni repercuten de manera uniforme, pese a que tengan un elemento en común: la desestabilización. Las crisis producen la eclosión de públicos específicos y varía la primacía concebida con antelación en cada grupo en los programas comunicativos convencionales (Bonilla et al., 2013).

Por ello, es necesario elaborar un plan que abarque cada una de las categorías tratando de minimizar riesgos y respuestas negativas de la ciudadanía. En la comunicación en crisis el silencio juega un papel negativo, denota inexperiencia, inseguridad y falta de liderazgo, por ende, influirá sobre los demás. Foucault (2002), planteaba que los itinerarios de la comunicación 
son la sustentación de un nódulo y de un centralismo del conocimiento, ya que los anclajes del poder están marcados por el juego de los signos.

Luhmann (1995), lo sintetizaba refiriéndose a que las diferenciaciones de sistemas establecidos estabilizan las posibilidades de reproducción al restringir las condiciones de comprensión de la comunicación y la idoneidad de los modos de comportamiento. Por ende, la manera cómo se transmite el contenido discursivo será determinante en la conducta del ciudadano, repercutiendo positivamente cuando acata recomendaciones en la inmediatez del caso o de manera negativa generando pánico y descontrol.

Se debe tener en cuenta que los mass media y las redes sociales divulgan el conocimiento de los expertos y la ciudadanía se suele apoderar de porciones de la información incorporándola en sus propias experiencias (Berger \& Luckmann, 1996). Sin embargo, la prominencia que las redes sociales han ganado como herramienta para la comunicación en crisis podría hacer que la información errónea, durante el reto de salud pública que ha supuesto la COVID-19, sea un fenómeno aún más complejo de tratar (van der Meer et al., 2020), ya que pese a que las redes sociales ejercen como elementos centrales para la aproximación a la información (Triviño, 2015), también hacen lo mismo cuando de desinformación se trata. El afán por viralizarse o ser tendencia, en casos malintencionados, conlleva a muchos usuarios a generar y replicar contenido sin fuentes fiables y constatables de información verídica. Recordemos que el usuario es prosumidor, produce y consume información ocupando un papel protagónico en el paradigma de la cultura participativa propiciado por la interactividad tecnológica (Aguaded \& Sánchez, 2013) y las redes sociales permiten la participación ciudadana de manera activa en la construcción de nuevas realidades presentes en la era de la información.

Para el tratamiento de las crisis por medio de las redes sociales, los contenidos gráficos son excelentes, considerando que gran parte de la ciudadanía no leen los textos, sino que únicamente leen los titulares (Catalan-Matamoros et al., 2020). Un aspecto importante a tener en cuenta es que, ante una crisis, la estrategia de comunicación deberá enfocarse en un público no especializado y deberá ser de carácter explicativo-propositivo. Además, resulta necesario que la misma pueda dotar a la ciudadanía de una competencia mediática idónea para la comprensión de los mensajes adecuadamente, ya que resulta necesario explicar las terminologías y los hallazgos científicos que ayudarían a calmar y asegurar a la población respecto a los pasos relevantes para reducir el virus (Mohamad \& Azlan, 2020). 


\section{La competencia mediática como elemento de la comunicación}

Una definición global de competencia mediática son el conjunto de habilidades necesarias para convivir con los medios y mantener una relación positiva con ellos (Gonzálvez-Pérez et al., 2015). El concepto de competencia mediática, se está adoptando desenfrenadamente y se encuentra en proceso de absorción de todas las habilidades en relación con la presencia de nuevos entornos culturales, relaciones sociopolíticas y componentes de la comunicación y la información. Todo esto, trae consigo cambios en las habilidades de aprendizaje, lectura, escritura y expresiones mediáticas (Garro-Rojas, 2019). En síntesis, la competencia mediática se define como propulsora de darle al ciudadano la capacidad y dotarlo de destrezas para saber elegir y reconocer la información que emiten los medios sociales. Es por eso, que es necesario cambios en la educación que propicie una ciudadanía con más competencias mediáticas (Delgado-Ponce \& Pérez-Rodríguez, 2012).

Según López y Aguaded (2015), para que la ciudadanía esté capacitada debe tener las competencias necesarias para transformar la emoción en reflexión y lo inverso, por lo que deberá ser competente y comprender la información para exponer razonamientos críticos de la comunicación que analiza diariamente; además, ser capaz de fabricar elementos comunicativos que inciten a la reflexión, que sean de fácil comprensión y comunicativamente eficaces, ya que no es noticia que los medios pueden influir drásticamente en las opiniones y comportamientos de los jóvenes (Mallia et al., 2020; Cho et al., 2020). Para Austin et al. (2020), la competencia mediática puede empoderar a los padres y mejorar a los jóvenes, haciéndoles perfeccionar gradualmente la utilización de los mass media para obtener mejor información.

Es de considerar que estamos en una época transitoria, aprendiendo a dejar de lado el condicionado, el despotismo y la exclusividad de la comunicación colectiva que acostumbró a creer en todo lo que veíamos y escuchábamos sin buscar veracidad en lo percibido, posicionándonos como ciudadanos pasivos y tímidos para exteriorizar nuestras respuestas, ya que no existía la posibilidad de interacción con los medios, ni otras vías donde poder manifestar la protesta o contestación (Orozco-Gómez et al., 2012).

Sin embargo pese a lo anterior expuesto, es menester reafirmar como aspecto negativo la universalidad de la desinformación, que pone en peli- 
gro a la democracia e intimida a la ciudadanía, a través de mensajes estratégicos que persiguen ganancias mercantiles e influir en comportamientos proselitistas presentes y futuros (Alcolea-Díaz et al., 2019), de ahí que, las nuevas tecnologías han generado que la naturaleza de la comunicación tradicional se volviera involutiva para el conglomerado, pero eficaces en la naturaleza misma de la comunicación como elemento interpersonal colectivo. Sin embargo, el nuevo horizonte tecnológico ha facilitado que la ciudadanía obtenga más herramientas para acceder a la información de distintas formas, provocando que la utilización de los medios digitales cambie el modo de producción de noticias drásticamente (Hernández-Serrano et al., 2017).

La exposición y uso prolongado de las diferentes redes sociales y servicios de comunicación, han cambiado nuestro modo de vida, no solo a nivel digital-mediático, sino también en lo que respecta a nuestras habilidades competitivas (competencia mediática), que sirven como elementos de nuestra propia comunicación. Bajo este enfoque, se requiere de una ciudadanía que tenga competencias que le proporcione habilidades como consumidor y creador de elementos audiovisuales, que sean conscientes de sus acciones y tengan las capacidades necesarias para realizar una crítica constructiva de la información (Gonzálvez-Pérez et al., 2015; García-Ruiz et al., 2014), en aras de la apropiación del pensamiento crítico por parte de la ciudadanía.

Finalmente, la competencia mediática como elemento imprescindible dentro de la comunicación, especialmente en este tiempo de crisis por la pandemia de la COVID-19, debe ser el desafío de todos los individuos susceptibles de las influencias constantes de la información, buscando adquirir un pensamiento crítico. Es decir, que se pueda crear y producir liderazgo desde las masas hacia los generadores de información, lo cual podría ser el principio de una detonación ascendente de valores, principios, moralidad, empoderamiento y creatividad (Pullaguari-Zaruma y Hernando-Gómez, 2019) de quienes un día fueron excluidos por no estar o pertenecer a una línea comunicativa.

\section{La estrategia de la comunicación en Twitter}

La utilización de Twitter en las estrategias de la comunicación aumenta cada día más. No cabe duda, que la diversidad en la convergencia y de los 
procesos tecnológicos acelerados, así como los cambios sociales, culturales, comunicativos, ambientales, políticos, entre otros, ha irrumpido en contemplar la comunicación de forma agresiva en los medios sociales; ocasionando que "grandes eventos" sean vislumbrados a través de las pantallas desde una mirada más crítica.

Las redes sociales ya están siendo explotadas para aprender, descubrir, buscar, almacenar y, sobre todo, compartir conocimientos, demostrando que son un vehículo eficiente para el aprendizaje social que podría agregarse como una herramienta competente para el aprendizaje formal. (López-Goñi \& Sánchez-Angulo, 2018, p. 1)

En el caso de Twitter, para Alvídrez y Franco-Rodríguez, (2016), permite conocer la relación de acciones diarias de la biografía pública, además su inmediatez lo convierte en una herramienta frecuente entre las personalidades públicas (políticos, burocráticos, religiosos, deportivos, exponentes culturales, entre otros) que buscan seducir a sus seguidores, crear una afinidad con ellos y movilizarlos a realizar una acción determinada.

Para Alonso-Muñoz y Casero-Ripollés (2018), la comunicación social es determinante, porque conserva una estrategia de comunicación inmediata que permite mantener contacto directo con la ciudadanía, que se convierte en los destinatarios de sus anuncios, y esto contribuye a burlar a los medios de comunicación, ya que estos son sus adversarios. La estrategia de Twitter puede entenderse como un canal para articular la hegemonía para quienes tienen el poder, ya que esta coyuntura se basa en relaciones intrapersonales y comunicación política (García-Carretero \& Pérez-Altable, 2017) como la estrategia angular de todo gobierno ante sus simpatizantes. En ocasiones, los interesados asumen roles protagónicos sobre debates o temas de interés mediáticos que interesa a la ciudadanía. En ese contexto, es significativo observar cómo ciertos grupos o individuos en el poder hacen uso de Twitter como una estrategia de comunicación masiva durante tiempos de crisis con el único objetivo de comunicar sus intereses. Según Tikka (2019), la comunicación en los medios sociales como Twitter pueden generar crisis en las sociedades.

Por otra parte, los acontecimientos en Twitter que se volvieron tendencias, debate y una alta expectativa, fue la información de salud que se ha convertido en tendencia como la más influyente para la sociedad, entre ellos jóvenes y minorías étnicas que tienen menos probabilidades de acceder a la 
información de salud a través de fuentes formales de noticias, entre otros recursos más tradicionales (Massey et al., 2016) son los más afectados. La comunicación en redes sociales puede ser sensible y crear angustia en personas vulnerables, varios estudios están poniendo mayor atención en la generación más joven (Burnap et al., 2017), ya que la influencia de los mensajes en Twitter es persuasiva cuando se trata de salud o participación política. Autores como Wang et al. (2020), sostienen que en un mundo globalizado la comunicación con los consumidores en las redes sociales puede ser oportuna y fundamental para sensibilizar y proceder a actuar.

En síntesis, la estrategia de la comunicación en Twitter es poliédrica; por un lado, puede ocuparse de la imagen y la reputación de personalidades públicas, políticas, deportivas, etc. (Gomes-Franco, 2016); mientras que por el otro, tratar de preservar la vida igualitaria de las personas cuando hablamos de efectos mundiales que atañen la salud. En Twitter, los límites de la comunicación se disuelven a medida que la noticia llega a sus pares y al público en general, incluidos los líderes de opinión, como periodistas y políticos (Jünger \& Fähnrich, 2020).

\section{Los países hispanohablantes}

El español es hablado por 400 millones de ciudadanos repartidos en 21 países (Argentina, Bolivia, Chile, Colombia, Costa Rica, Cuba, Ecuador, España, Guatemala, Guinea Ecuatorial, Honduras, México, Nicaragua, Panamá, Paraguay, Perú, Puerto Rico, República Dominicana, Uruguay y Venezuela). Es hablado también en Andorra, Estados Unidos, Filipinas y en Sahara Occidental, aunque no son los idiomas oficiales en esos países (Lyons, 2020).

\section{Metodología}

Esta investigación se desarrolló de acuerdo a los siguientes objetivos:

- Analizar la comunicación gubernamental y competencia mediática de los presidentes hispanohablantes la semana previa a la declaración de pandemia. 
- Diseñar una plantilla para la medición de la competencia mediática en Twitter.

- Observar y comparar la evolución del brote hasta la declaración de pandemia y su tratamiento por los presidentes.

Para el desarrollo de esta investigación se utilizó una metodología cuantitativa. Gracias a la minería de datos en la red social Twitter, con una muestra 7650 tuits, de los que 884 tuits fueron publicados en 22 cuentas de los presidentes hispanohablantes y 6766 tuits analizados de los mass media de los países hispanohablantes durante la semana previa a la declaración de pandemia por la OMS.

Se establece el periodo de estudio del 4 al 11 de marzo, puesto que el día antes la OMS invitó a los gobiernos y a la Pandemic Supply Chain Network para impulsar la producción y asegurar los suministros para los países en riesgo y los gravemente afectados por una posible pandemia.

Tras la recogida de los tuits a través de diversos algoritmos de búsquedas avanzadas y de herramientas como Metricool, TweetStats, Tweet Topic Explorer y Burrrd se analizaron los datos extraídos de la muestra con el objeto de establecer el nivel de comunicación gubernamental y competencia mediática durante los días previos a la declaración de la pandemia.

\section{Resultados}

\section{Observación de la competencia mediática de los presidentes hispanohablantes}

Para realizar la observación y estudio de la competencia mediática de las cuentas de Twitter de los presidentes con base en las dimensiones desarrolladas por Ferrés y Piscitelli (2012), diseñamos un modelo de medición de la competencia mediática en Twitter utilizando diversos índices de cálculo con el objeto de lograr datos objetivos en las mediciones de cada una de las dimensiones. En la tabla 1 se puede ver la relación de las dimensiones establecidas para la competencia mediática y los índices que establecimos en nuestro modelo de estudio. 


\section{Tabla 1}

Plantilla para estudiar la competencia mediática en Twitter

\begin{tabular}{|l|l|}
\hline \multicolumn{1}{|c|}{ Dimensiones por Ferrer y Piscitelli } & Parámetros de medida de las dimensiones \\
\hline Lenguajes & Índice Flesch-Kincaid \\
\hline Interacción & Engagement \\
\hline Tecnología & Soporte audiovisual \\
\hline Producción y difusión & Índice Link-Hashtag \\
\hline Ideología y valores & Índice de autenticidad \\
\hline Estética & Agenda building. Compromiso \\
\hline
\end{tabular}

Fuente: Elaboración propia.

\section{Índice Flesch-Kincaid: facilidad lectura}

Analizamos las cuentas de los presidentes de cada uno de los países hispanohablantes para recabar datos que nos facilitaron la labor para realizar el análisis de la competencia mediática de estos. Para ello, utilizamos diversas herramientas para conocer el número de tuits y retuits que los presidentes realizaron durante el periodo analizado, los dispositivos electrónicos desde dónde se hacían las publicaciones, el promedio de palabras utilizadas y la valoración de la complejidad de texto utilizado mediante la prueba de legibilidad Flesch-Kincaid, la cual indica el nivel de facilidad de lectura en una escala de 0-100. (Siendo 0-30 lectura muy difícil que solo entenderían graduados universitarios hasta llegar a la de 90-100 de lectura fácil que entenderían estudiantes de once años sin esfuerzo).

Cuatro de las cuentas tenían un índice Flesch entre 30-50 siendo consideradas de difícil lectura, el resto de cuentas presentaban un índice Flesch inferior a 30 siendo consideradas de muy difícil lectura, es decir, que prácticamente todos los mensajes de textos presentan dificultades en su lectura entre sus seguidores (gráfico 1). Realizando un promedio entre todas las cuentas, diez de ellas estarían incluso por debajo del promedio de todas las cuentas, lo que las convertiría en las cuentas con más dificultad de lectura de todas. 


\section{Gráfico 1 \\ Índice Flesch-Kincaid de facilidad de lectura de los tuits}

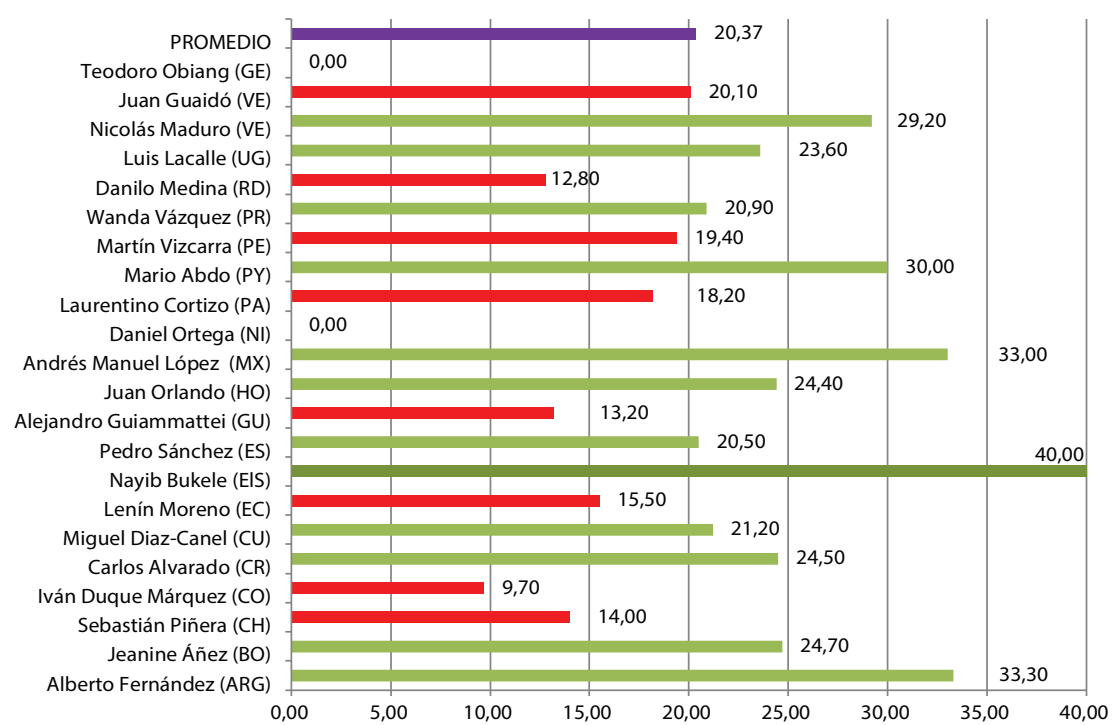

Fuente: Elaboración propia.

\section{Engagement: interacción y compromiso}

Por otro lado, hemos medido el compromiso y la interacción de las cuentas con sus seguidores, lo que se denomina el engagement del usuario de Twitter, y para ello, se utilizó una fórmula estándar: la suma del número de menciones, retuits y likes divididos por el número de seguidores en total, y finalmente multiplicado por 100. Como podemos observar en el gráfico 2 la media del engagement se situó en 129.53, donde solo las cuatro cuentas de los presidentes de Argentina, Cuba, Ecuador y una de los presidentes de Venezuela se situaron con unos valores muy por encima de la media. El resto de cuentas se podría indicar que tienen un engagement muy bajo con respecto a la media de todos, por lo que se entiende que tiene poca interacción con los miembros de su comunidad de seguidores. 


\section{Gráfico 2}

\section{Engagement de las cuentas de los presidentes}

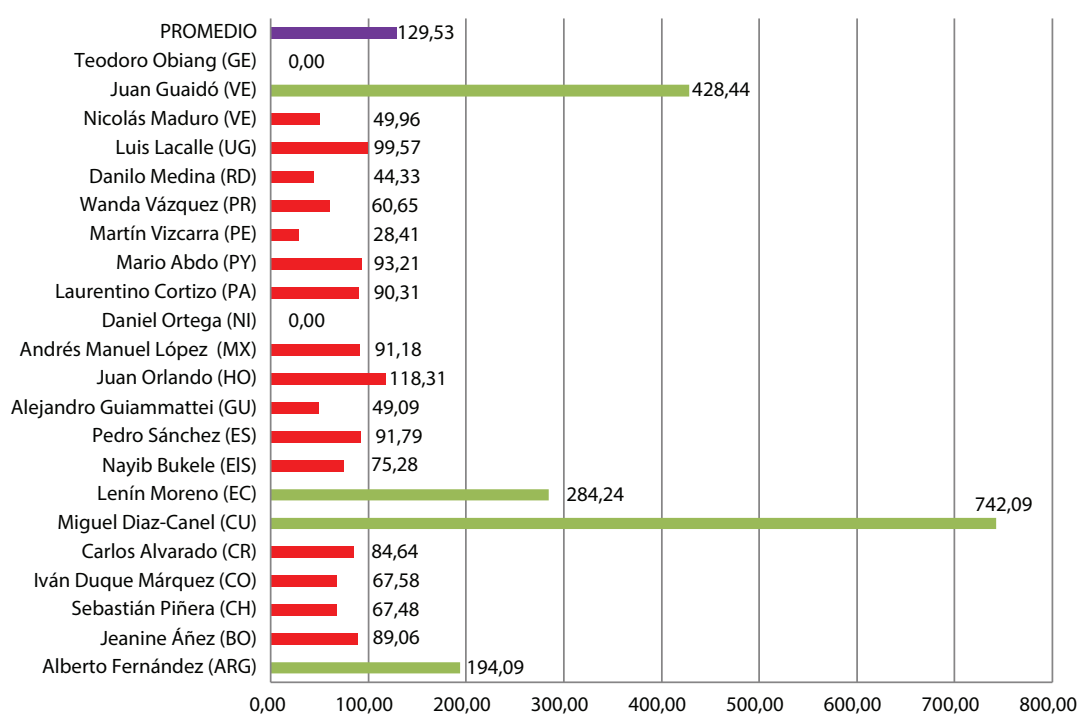

Fuente: Elaboración propia.

\section{Soporte audiovisual: tecnología}

Para el análisis de la competencia tecnológica, la cual es bastante amplia, hemos tenido en cuenta para la medición en las publicaciones en Twitter, aquellas que presentan en sus mensajes contenidos audiovisuales, ya sean fotografías, vídeos o infografías, puntuando aquellas que sí tienen ese contenido y no contemplando puntaje en aquellos tuits que solo presentan texto plano sin enriquecimiento del mismo. En el gráfico 3 podemos observar cómo de los 22 presidentes, ocho de ellos presentan material audiovisual en todas sus publicaciones, otros tres presentan material audiovisual en la mayoría de sus publicaciones, seis de ellos no incluían ningún material audiovisual y cinco no hicieron ninguna publicación sobre COVID-19, por lo que no pudieron ser evaluados. La media que presentan las cuentas es de un 47.22 de publicaciones con contenido audiovisual. 


\section{Gráfico 3}

\section{Soporte audiovisual de las cuentas de los presidentes}

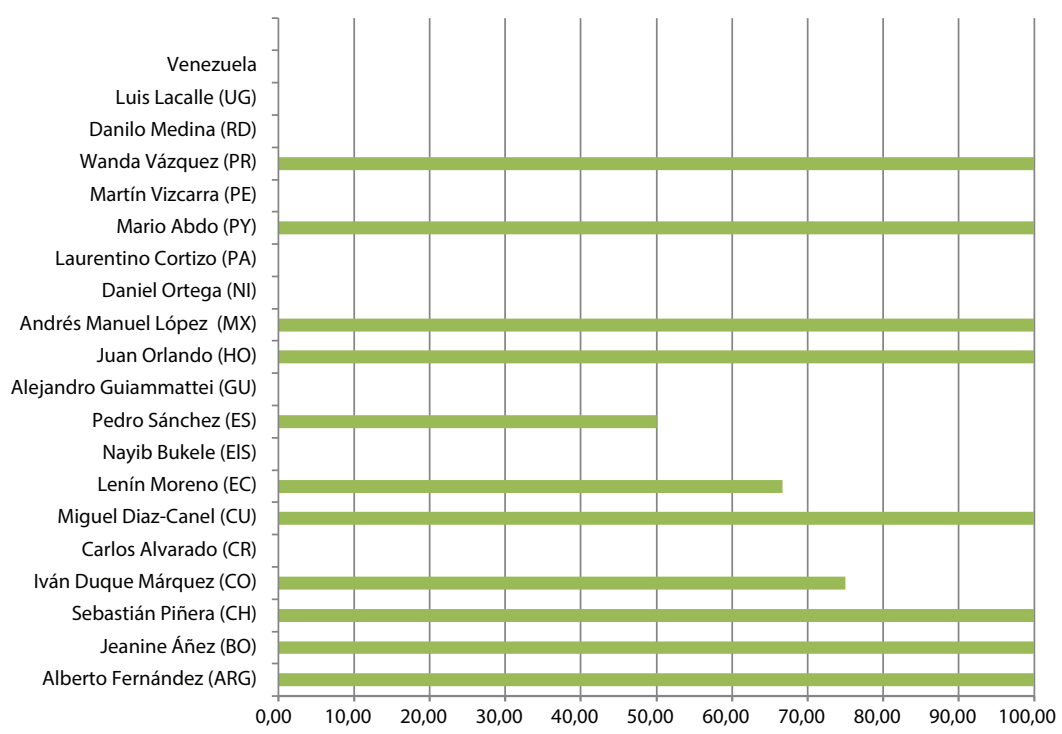

Fuente: Elaboración propia.

\section{Índice Link-Hashtag: producción y difusión}

Para analizar el correcto uso de publicación, tuvimos en cuenta dos factores: el índice de enlaces en publicaciones y el índice de utilización de hashtags. El promedio de los enlaces por tuit publicado, indica la probabilidad de que el usuario sea una fuente de información para otros, y el promedio de inclusión de hashtags nos indica la probabilidad de que los tuits del usuario se encuentren en una búsqueda (gráfico 4). Como podemos comprobar en la figura siguiente los niveles de producción y difusión eran muy bajos para todas las cuentas, ninguna de ellas llegó a una media de un enlace por tuit, y haciendo un promedio, quince cuentas estaban por debajo del mismo que se situó en 0.14 enlaces. En cuanto al nivel de hashtag, solo una de las cuentas superó más de dos por tuit, situándose la media en 0.46 , de las que trece cuentas se situaron por debajo de la misma a pesar de ser un promedio muy bajo. 


\section{Gráfico 4 \\ Índices de producción y difusión de los presidentes}

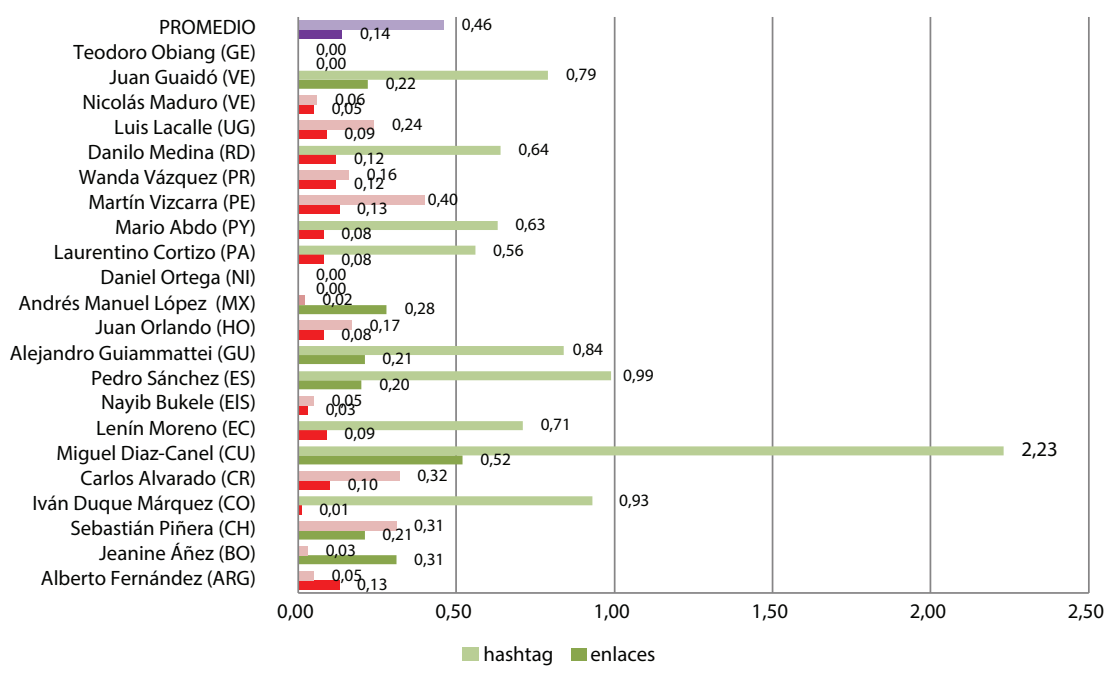

Fuente: Elaboración propia.

\section{Índice de autenticidad: valores}

Para analizar la autenticidad en Twitter, es decir, el valor que hace referencia al porcentaje de los mensajes que son emitidos por el propio usuario desde su dispositivo electrónico. Fue una de las medidas más complejas, pero teniendo en cuenta los usuarios a los que estábamos midiendo, determinamos como índice el dispositivo móvil del que disponían y desde el que se realizaron el número mayor de publicaciones. De los 19 presidentes analizados, 16 utilizan un iPhone, dos utilizaron un Smartphone con Android, y uno utilizó un iPad. De diez de las cuentas se aprecia que se usó software de programación de tuits, y en siete de ellos se aprecia que utilizaron un dispositivo móvil de otro usuario (gráfico 5).

Solo seis de las 19 cuentas analizadas indican que los mensajes son del usuario propietario de la cuenta, mientras que se colige que en cinco de las cuentas la mayoría de los tuits publicados no provienen de sus dispositivos y por ello tampoco de su propia creatividad. 


\section{Gráfico 5 \\ Índices de autenticidad en la publicación de los presidentes}

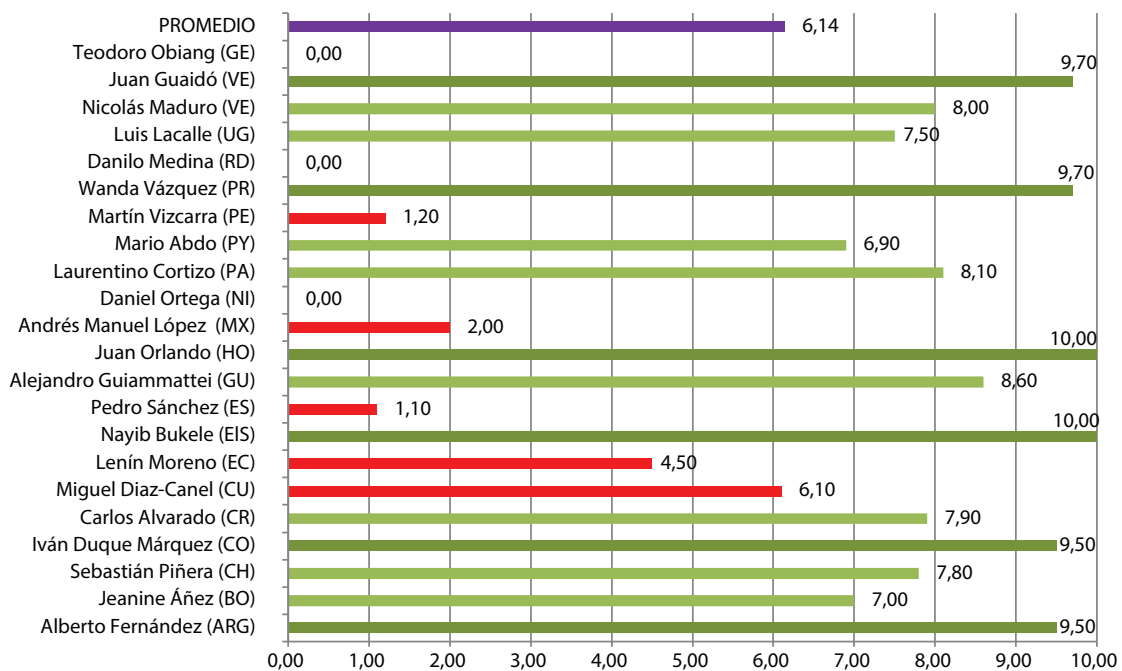

Fuente: Elaboración propia.

\section{Agenda Building y Agenda Setting: nivel de compromiso durante la COVID-19}

Estos cálculos se realizaron teniendo en cuenta el número total de tuits publicados y aquellos que estaban relacionados únicamente con la COVID-19. Para dichas conjeturas tuvimos en cuenta dos factores: la presión social emitida desde los mass media y las comunicaciones realizadas por los presidentes, debido a que la Agenda Building (construcción de la agenda mediática) se concentra en la cimentación de las noticias y los componentes que la respaldan (Aruguete, 2017).

A finales del 2019, los mass media comenzaron a publicar noticias sobre el coronavirus, intensificando dichas publicaciones a partir de enero de 2020, al momento de declararse una masificación de los casos por el gobierno chino en Wuhan y con mayor frecuencia en el momento de traspasar las fronteras de China. Después de la primera señal importante de una posible pandemia el 3 de marzo, hasta su declaración oficial por parte de la OMS el 11 de marzo, los 
medios de comunicación intensificaron el volumen de noticias relacionadas con la COVID-19 para un total de 1887 publicaciones frente 6766 de temas varios, lo que representaba un $27.89 \%$ de impacto total.

Entendemos que los mass media actúan con mucha influencia en toda la ciudadanía, y los presidentes de los países hispanohablantes son de igual forma influidos por estos. Sin embargo, en el nivel de presencia de la comunicación gubernamental en sus publicaciones por medio de Twitter no se observó que fuese en la misma línea que los medios de comunicación. El número total de publicaciones por los presidentes, durante el periodo de estudio, fue de un total de 884 tuis de los que tan solo 92 estaban relacionados con la COVID-19, lo que representaba un nivel de impacto del $10.41 \%$, que como podemos observar estaba muy por debajo del nivel de impacto de los mass media, lo que creó un desajuste en la Agenda Building de los presidentes hispanohablantes. En el gráfico 6 se observan las diferencias entre el nivel de impacto de la prensa y de los presidentes pormenorizados por cada país hispanohablante.

\section{Gráfico 6 \\ Impactos de publicaciones de medios de comunicación y presidentes}

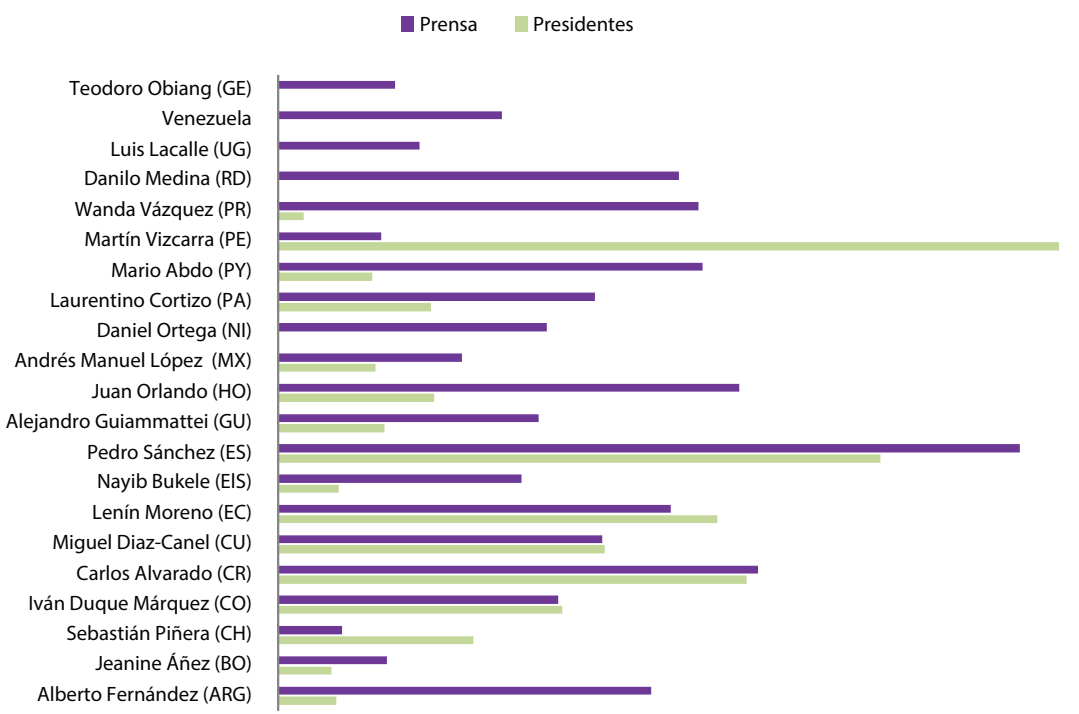

Fuente: Elaboración propia. 
Como se colige, tan solo dos presidentes presentaron datos por encima de la prensa de su país y de la media de todos los perfiles estudiados, y cuatro de ellos tenían un buen equilibrio con la Agenda Building. Catorce cuentas presentaron datos por debajo de la Agenda Building y cuatro de ellas ni siquiera tenían datos relacionados con esta.

\section{Discusión}

Tras la aparición de Internet y las redes sociales, a la alfabetización mediática e informacional se le añaden nuevos componentes de alfabetización digital. Esto se debe a que los mass media están digitalizando su comunicación, por lo que se hace necesario poseer mejores competencias digitales (Delgado-Ponce \& Pérez-Rodríguez, 2012).

Los mensajes tienen un contenido basado en la interpretación subjetiva del mismo en distintos contextos, así como una explicación de estos cuando es posible (Conde et al., 2019). Twitter es un medio de presión en ambas vías, por un lado, tenemos a los ciudadanos inconformes respecto a determinadas acciones o discursos incongruentes por parte de la gobernanza y por el otro, al gobierno que trata de exponer y afianzar un respaldo a sus procesos en situaciones normales como en las de crisis.

Con el modelo de estudio de la competencia mediática diseñado en esta investigación, los parámetros que medimos fueron los de: índice Flesch-Kincaid, para analizar la dimensión de lenguajes; el engagement, para la dimensión de interacción; el soporte audiovisual, para la de tecnología; el índice link-hashtag, para la de producción y difusión; el índice de autenticidad, para la de ideología y valores; y finalmente, la agenda building, para la de ética.

En cuanto al índice Flesch-Kincaid: ninguna de las cuentas analizadas superó el índice mínimo para considerar una buena utilización del lenguaje en el contenido de las publicaciones. Por lo que concluimos, que la dimensión de lenguajes no es superada por todas las cuentas de los presidentes, indicando la necesidad de mejorar el contenido de sus tuits para que un espectro más amplio de población pueda entender más fácilmente el mensaje.

El engagement de las cuentas analizadas: tan solo cuatro de ellas superaron el promedio, el resto quedaron con unos niveles muy por debajo de la media. Esto nos conlleva a concluir que la dimensión de interacción con su comunidad es deficiente y debe ser trabajada para mejorar los índices. Posi- 
blemente el bajo índice en la interacción esté relacionado con el uso de un lenguaje medianamente complejo para la mayoría de la ciudadanía.

Con respecto al soporte audiovisual utilizado por las cuentas, restando aquellas cuentas que no hicieron publicaciones durante el periodo analizado, concluimos que se mantiene por encima de la media. La dimensión tecnología presenta unos datos bastante positivos en su mayoría.

El índice link-hashtag: fue bastante bajo para todas las cuentas analizadas, concluimos que la dimensión de producción y difusión es bastante deficiente en general, lo que convertiría estas cuentas en fuentes no fidedignas de información para otros usuarios, ni que tampoco aparezcan de forma mayoritaria en las búsquedas de Twitter.

El índice de autenticidad o veracidad: el contenido de las publicaciones proviene del titular de la cuenta fue por regla general muy alto, salvo unas pocas cuentas que se intuyen que el contenido mayoritariamente no provenía del titular de la misma. Colegimos, indicando que la dimensión ideológica y valores, que la mayoría de las cuentas la aprueban con índices muy buenos.

El contenido de las publicaciones de los presidentes no iba en consonancia con la Agenda Building; es decir, el nivel de compromiso y ética de las cuentas durante la COVID-19 no fue correcto. Tan solo cinco de las veintidós cuentas analizadas tenían competencias mediáticas en la dimensión de ética.

Constatamos que durante el periodo escogido las cuentas analizadas, no presentan las competencias mediáticas adecuadas en cinco de las seis dimensiones. Sugerimos que los presidentes hispanohablantes necesitan estudiar profundamente su comunicación gubernamental vía Twitter, pues necesitan mejorar de forma notable su competencia mediática en la comunicación con la ciudadanía.

\section{Bibliografía}

Aguaded, J. I., \& Sánchez, J. (2013). El empoderamiento digital de niños y jóvenes a través de la producción audiovisual. AdComunica: Revista Científica de Estrategias, Tendencias e Innovación en Comunicación, 2013(5), 175-196. https://doi.org/10.6035/2174-0992.2013.5.11

Alcolea-Díaz, G., Reig, R., \& Mancinas-Chávez, R. (2019). Currículo de Alfabetización Mediática e Informacional de la UNESCO para profesores desde la perspectiva de la Estructura de la Información. Comunicar, 62, 103114. https://doi.org/10.3916/C62-2020-09 
Alonso-Muñoz, L., \& Casero-Ripollés, A. (2018). Communication of European populist leaders on twitter: Agenda setting and the "more is less" effect. Profesional de La Informacion, 27(6), 1193-1202. https://doi.org/10.3145/ epi.2018.nov.03

Alvídrez, S., \& Franco-Rodríguez, O. (2016). Estilo comunicativo súbito en Twitter : efectos sobre la credibilidad y la participación cívica. Comunicar, 47(2), 89-97. https://doi.org/10.3916/C47-2016-09

Aruguete, N. (2017). Agenda building. Revisión de la literatura sobre el proceso de construcción de la agenda mediática. Signo y Pensamiento, 36(70), 3854. https://doi.org/10.11144/Javeriana.syp36-70.abrl

Austin, E. W., Austin, B. W., \& Kaiser, C. K. (2020). Effects of Family-Centered Media Literacy Training on Family Nutrition Outcomes. Prevention Science, 21(3), 308-318. https://doi.org/10.1007/s11121-020-01101-X

Bauman, Z., \& Bordoni, C. (2016). Estado de crisis (1st ed.). Paidós.

Berger, P., \& Luckmann, T. (1996). Modernidad, pluralismo y crisis de sentido (1st ed.). Paidós.

Bonilla, C., Gil, P., Alcázar, P., Arribas, A., García, Y., Lorenzo, M., Ricón, J. A., García, S., Benassini, C., Vidal, F., Hernández, H.G., Rodríguez, L.A., Islas, O., \& Hitrón, L.M. (2013). Investigando la comunicación en crisis (1st ed.). Tecnológico de Monterry. https://bit.ly/36Nrx0Z

Burnap, P., Colombo, G., Amery, R., Hodorog, A., \& Scourfield, J. (2017). Multiclass machine classification of suicide-related communication on Twitter. Online Social Networks and Media, 2, 32-44. https://doi.org/10.1016/j. osnem.2017.08.001

Canel, M. J., \& Sanders, K. (2011). Government Communication. In The International Encyclopedia of Communication (pp. 1-5). https://doi. org/10.1002/9781405186407.wbiecg033

Catalan-Matamoros, D., Nascimento, B. G. do, \& Langbecker, A. (2020). El contenido visual publicado en prensa durante una crisis sanitaria: El caso del Ébola, España 2014. Interface - Comunicação, Saúde, Educação, 24, 1-18. https://doi.org/10.1590/interface.190271

Cho, H., Song, C., \& Adams, D. (2020). Efficacy and Mediators of a Web-Based Media Literacy Intervention for Indoor Tanning Prevention. Journal of Health Communication, 25(2), 105-114. https://doi.org/10.1080/108107 30.2020.1712500

Conde, M., Prada-Espinel, O., \& Pullaguari, K. (2019). Comparativa de la competencia mediática en comunicación política vía Twitter durante las 
campañas electorales de candidatos presidenciales en España, Ecuador y Colombia. Contratexto, 32, 41-70. https://doi.org/10.26439/contratexto2019.n032.4606

Coombs, T. (2007). Protecting Organization Reputations During a Crisis : The Development and Application of Situational Crisis Communication Theory,10(3), 163-176. https://doi.org/10.1057/palgrave.crr.1550049

Coombs, T. (2010). Parameters for Crisis Communication. In The handbook of Crisis Comunication (1st ed.). Blackwell Publishing.

Delgado-Ponce, Á., \& Pérez-Rodríguez, M. A. (2012). De la competencia digital y audiovisual a la competencia mediática: dimensiones e indicadores. Comunicar, 20(39), 25-33. https://doi.org/10.3916/C39-2012-02-02

Ferrés, J., \& Piscitelli, A. (2012). La competencia mediática: propuesta articulada de dimensiones e indicadores. Revista Comunicar, XIX(38), 75-82. https://doi.org/10.3916/C38-2012-02-08

Foucault, M. (2002). Vigilar y castigar. 1st ed. Siglo XXI.

García-Carretero, L., \& Pérez-Altable, L. (2017). Barcelona en comú on twitter. Analyzing the electoral communication of the confluence during the 2015 council election. Profesional de la informacion, 26(5), 871-883. https://doi.org/10.3145/epi.2017.sep.09

Garro-Rojas, L. (2019). Alfabetización mediática en América Latina. Revisión de literatura: temas y experiencias. Revista Educación, 44, 22. https://doi. org/10.15517/revedu.v44i1.37708

Gomes-Franco, F. (2016). Estrategias comunicativas de escritores españoles en Twitter. Revista Mediterránea de Comunicación, 7(1), 231-254. https:// doi.org/10.14198/medcom2016.7.1.15

Gonzálvez-Pérez, V., García-Ruiz, R., \& Aguaded Gómez, J. I. (2015). La competencia mediática como reto para la educomunicación: instrumentos de evaluación. Cuadernos.Info, 35, 15-27. https://doi.org/10.7764/cdi.35.623

Heath, R. (2010). Crisis Communication: Defining the Beast and De-marginalizing Key Publics. In The handbook of Crisis Comunication (1st ed., p. 737). Blackwell Publishing.

Hernández-Serrano, M. J., Renés-Arellano, P., Graham, G., \& Greenhill, A. (2017). From prosumer to prodesigner: Participatory news consumption. Сотиnicar, 25(50), 77-87. https://doi.org/10.3916/C50-2017-07

Jünger, J., \& Fähnrich, B. (2020). Does really no one care? Analyzing the public engagement of communication scientists on Twitter. New Media and Society, 22(3), 387-408. https://doi.org/10.1177/1461444819863413 
López-Goñi, I., \& Sánchez-Angulo, M. (2018). Social networks as a tool for science communication and public engagement: Focus on Twitter. FEMS Microbiology Letters, 365(2). https://doi.org/10.1093/femsle/fnx246

López, L., \& Aguaded, M. C. (2015). Teaching media literacy in colleges of education and communication. Comunicar, 22(44), 187-195. https://doi. org/10.3916/C44-2015-20

Luhmann, N. (1995). Social Systems (1st ed.). Stanford University Press.

Lyons, D. (2020). ¿Cuántas personas hablan español en el mundo y en qué países se habla? Babbel. https://bit.ly/2WmjSlv

Mallia, L., Chirico, A., Zelli, A., Galli, F., Palombi, T., Bortoli, L., Conti, C., Diotaiuti, P., Robazza, C., Schena, F., Vitali, F., Zandonai, T., \& Lucidi, F. (2020). The Implementation and Evaluation of a Media Literacy Intervention About PAES Use in Sport Science Students. Frontiers in Psychology, 11(March), 1-10. https://doi.org/10.3389/fpsyg.2020.00368

Massey, P. M., Leader, A., Yom-tov, E., Budenz, A., Fisher, K., \& Klassen, A. C. (n.d.). recopilación de datos para cuantificar la comunicación de la vacuna contra el virus del papiloma humano en Twitter.

Mohamad, E., \& Azlan, A.A. (2020). COVID-19 and Communication Planning for Health Emergencies. Jurnal Komunikasi: Malaysian Journal of Communication, 36(1).

Molina, S. (2004). Comunicación gubernamental: ¿encanto o desencanto? Revista Mexicana de Ciencias Políticas y Sociales, 46(190), 31-45. http://dx.doi. org/10.22201/fcpys.2448492xe.2004.190.42432

Orozco-Gómez, G., Navarro-Martínez, E., \& García-Matilla, A. (2012). Desafíos educativos en tiempos de auto-comunicación masiva: la interlocución de las audiencias. Comunicar: Revista Científica Iberoamericana de Comunicación y Educación, 38, 67-74.

Pullaguari-Zaruma, K. P., \& Hernando-Gómez, A. (2019). Politización mediática de la justicia en Ecuador. Estudio de caso: Ecuavisa. Revista Humanidades, 9(2), 1-23. https://doi.org/10.15517/h.v9i2.37659

Reyes, M., O’quínn, J., Morales, J., \& Rodríguez, E. (2011). Reflexiones sobre la comunicación política. Espacios Públicos, 14(30). https://bit.ly/3pWov22

Riorda, M. (2011). comunicación gubernamental Mario Riorda. POLITAI, 2(3). https://bit.ly/3pSb2bp

Tikka, M. (2019). Ritualisation of Crisis Communication. Nordicom Review, 40(1), 105-120. https://doi.org/10.2478/nor-2019-0006 
Triviño, A. (2015). Tecnología, redes sociales, política y periodismo. ¿pluralidad informativa o efecto bumerán? Cuadernos.Info, 36, 191-205. https://doi. org/10.7764/cdi.36.647

van der Meer, G.L.A., T., \& Yan, J. (2020). Seeking Formula for Misinformation Treatment in Public Health Crises: The Effects of Corrective Information Type and Source. Health Communication, 35(5), 560-575. https://doi.org /10.1080/10410236.2019.1573295

Wang, R., Huang, S., \& Pérez-Ríos, N. G. (2020). Multinational Luxury Brands' Communication Strategies on International and Local Social Media: Comparing Twitter and Weibo. Journal of International Consumer Marketing, O(0), 1-11. https://doi.org/10.1080/08961530.2019.1710736

\section{Anexos}

Los anexos de esta investigación se pueden ver en el siguiente enlace: https://bit.ly/2TcK9At o haciendo una captura en el código QR que se acompaña, que permitirá al lector visitar los anexos desde su Smartphone o Tablet de forma automática.

\section{Figura 1}

Anexos de la investigación

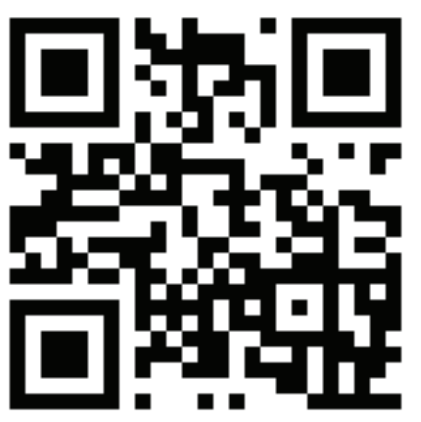

Elaboración propia.

Fecha de envío: 2020/10/20; Fecha de aceptación: 2021/02/01;

Fecha de publicación: 2021/03/01 